\title{
Tempo letal e período residual de inseticidas a ninfas e adultos do percevejo marrom da soja (Euschistus heros) (Fabricius, 1794) (Heteroptera: Pentatomidae)
}

\author{
Lethal and residual time of insecticides to nymphs and adults of the brown stink bug (Euschistus \\ heros) (Fabricius, 1794) (Heteroptera: Pentatomidae) \\ Tiempo letal y período residual de insecticidas para ninfas y adultos de la chinche parda \\ (Euschistus heros) (Fabricius, 1794) (Heteroptera: Pentatomidae)
}

Recebido: 21/05/2021 | Revisado: 29/05/2021 | Aceito: 29/05/2021 | Publicado: 03/06/2021
Larissa Alves de Castro Jocarelli Rossini ORCID: https://orcid.org/0000-0002-0324-6140
Universidade Estadual Paulista, Brasil E-mail: larissajrossini@gmail.com
Abraão Almeida Santos
ORCID: https://orcid.org/0000-0001-5284-3294 Universidade Federal de Viçosa, Brasil E-mail: abraaoufs@gmail.com
Marcelo Coutinho Picanço
ORCID: https://orcid.org/0000-0002-1294-6210 Universidade Federal de Viçosa, Brasil E-mail: picanco@ufv.br

\begin{abstract}
Resumo
O percevejo marrom (Euschistus heros) é considerado a principal espécie praga da soja no Brasil, com ampla distribuição geográfica predominando nas regiões Centro-Oeste e norte do Paraná. Além disso, esta praga apresenta tolerância aos inseticidas químicos utilizados para o seu controle. Deste modo, o objetivo desse trabalho foi avaliar o tempo letal e o período residual de inseticidas em ninfas e adultos do percevejo-marrom. Foram utilizados os inseticidas lambda-cialotrina + tiametoxam, imidacloprido, imidacloprido + bifentrina, bifentrina e acefato, e em sequência submetidos as ninfas e os adultos de percevejo marrom a alimentação foram submetidos aos inseticidas acima para determinação do tempo letal e período residual dos produtos. Os inseticidas Imidacloprido + Bifentrina e Lambda-cialotrina + Tiametoxam são mais eficientes para o controle de ninfas e adultos de E. heros por apresentarem menor tempo letal e maior período residual.
\end{abstract}

Palavras-chave: Percevejos; Controle químico; Piretróide; Neonicotinóide; Organofosforado.

\begin{abstract}
The brown stink bug is considered the main soybean pest species in Brazil, as it has a wide geographic distribution predominating in the midwest and northern regions of Paraná and greater tolerance to chemical insecticides. Thus, this study aimed to evaluate the lethal time and residual period of insecticides in nymphs and adults of the stink bug. The insecticides lambda-cyhalothrin + thiamethoxam, imidacloprid, imidacloprid + bifenthrin, bifenthrin and acephate were used, and in sequence the nymphs and the adult stink bug were subjected to these insecticides to determine the lethal time and residual period. The insecticides Imidacloprido + Bifentrina and Lambda-cyialotrina + Tiametoxam are the most efficient for the control of nymphs and adults of E. heros because they have less lethal time and longer residual period.
\end{abstract}

Keywords: Bedbugs; Chemical control; Pyrethroid; Neonicotinoid; Organophosphate.

\section{Resumen}

La chinche parda es considerada la principal plaga de la soja en Brasil, ya que tiene una amplia distribución geográfica predominando en las regiones del Medio Oeste y norte de Paraná y mayor tolerancia a los insecticidas químicos. El objetivo de este trabajo fue evaluar el tiempo letal y período residual de insecticidas en ninfas y adultos de la chinche apestosa. Se utilizaron los insecticidas lambda-cihalotrina + tiametoxam, imidacloprid, imidacloprid + bifentrina, bifentrina y acefato, y en secuencia las ninfas y la chinche adulta fueron sometidas a los insecticidas anteriores para determinar el tiempo letal y período residual de los productos. Los insecticidas Imidacloprido + Bifentrina y Lambda-cyialotrina + Tiametoxam son los más eficientes para el control de ninfas y adultos de E. heros porque tienen menos tiempo letal y mayor período residual.

Palabras clave: Chinches; Control químico; Piretroide; Neonicotinoide; Organofosfato. 


\section{Introdução}

A cultura da soja (Glycine max) tem grande importância para a economia brasileira, sendo o Brasil o maior produtor mundial, com uma área plantada de aproximadamente 36 milhões de hectares (Conab, 2020). Apesar de ser cultivada em todas as regiões brasileiras, a produtividade desta cultura é frequentemente reduzida devido ao ataque de inúmeros insetos-praga, que ocorrem durante todo o ciclo de cultura.

Dentre as principais pragas responsáveis pelas perdas de produtividade na soja, o complexo de percevejos pentatomídeos têm papel relevante, principalmente pela capacidade de danos diretos ao produto comercializável (CorrêaFerreira \& Azevedo, 2002; Panizzi et al., 2012; Moscardi et al., 2012). Estes insetos também causam danos indiretos devido ao ataque, como a abertura para doenças fúngicas (Galileo \& Heinrichs, 1978).

O percevejo marrom (Euschistus heros) (Fabricius, 1794) (Heteroptera: Pentatomidae) é responsável pela redução de até 30\% na produção de soja (Vivan \& Degrande, 2011), gerando consideráveis danos econômicos para os sojicultores. O percevejo marrom é considerado a principal espécie praga da soja no Brasil, por apresentar ampla distribuição de ocorrência e maior tolerância aos inseticidas químicos (Sosa-Gómez et al., 2009; Panizzi, 2015). Essa espécie de percevejo aumentou em grande proporção nos últimos anos em todo território nacional, sendo dominante na faixa entre a região Centro-Oeste e norte do Paraná. Há também registros de sua incidência em outras áreas produtoras de soja do país, como no estado do Maranhão, onde o percevejo marrom correspondeu à aproximadamente 90\% dos percevejos coletados (Corrêa-Ferreira et al., 2009; Panizzi et al., 2012).

Por ser a espécie mais abundante e predominante nas áreas agrícolas do Brasil (Krinski et al., 2013), estes percevejos ocorrem na cultura da soja em todas as fases de desenvolvimento, embora sejam prejudiciais a partir do início da formação das vagens até a maturação dos grãos (Zambiazzi et al., 2012). Suas populações iniciais são evidenciadas em geral em V6 e os picos populacionais são nas fases reprodutivas da soja (Borges et al., 2011). O dano ocorre quando os adultos e as ninfas se alimentam de grãos em desenvolvimento causando perdas de produtividade, além de perdas da qualidade principalmente em campos de produção de semente que altera padrões de vigor e germinação (Sosa-Gómez \& Panizzi, 1995; Ferrazza et al., 2020), e em altos ataques as perdas podem ser superiores a 50\% (Vivan \& Degrande, 2011).

Para reduzir os prejuízos causados pelo percevejo marrom na cultura da soja, o controle químico tem sido a medida mais utilizada pelos agricultores, podendo ser realizado por meio de aplicações preventivas de inseticidas sintéticos (SosaGómez \& Silva, 2010; Bueno et al., 2013). Portanto, a avaliação de eficácia e período residuais de inseticidas a esta praga são de grande importância uma vez que avaliando as eficiências ao longo do tempo residual pode contribuir para a escolha da melhor molécula.

\section{Metodologia}

A colônia de percevejo marrom foi originaria de adultos coletados em lavouras de soja (19 ${ }^{\circ} 46^{\prime} 15,58^{\prime \prime} \mathrm{S}, 54^{\circ}$ $60^{\prime} 83,87^{\prime \prime}$ W) no ano de 2017, no município de São Gabriel do Oeste - MS. Posteriormente, foi estabelecida uma criação massal no laboratório, em caixas plásticas $(20 \times 40 \times 14 \mathrm{~cm})$ e mantidos em sala climatizada $\left(25^{\circ} \mathrm{C}\right.$, UR $65 \pm 10 \%$ e fotofase de 16 horas). Para alimentação de ninfas e adultos foi fornecido um mix de grãos contendo vagens verdes de feijão (Phaseolus vulgaris), soja (G. max), amendoim (Arachis hypogaea) e girassol (Helianthus annuиs).

A higienização das caixas de criação e o fornecimento de alimento foram realizados três vezes por semana. Para a oviposição foram utilizados algodões hidrófilos e voil. Os ovos foram coletados e transferidos em placas de petri com algodão umedecido para eclosão $\left(25^{\circ} \mathrm{C}\right.$, UR $65 \pm 10 \%$ e fotofase de 16 horas).

Para a determinação do tempo letal dos produtos, foi realizado experimento utilizando os tratamentos apresentados na Tabela 1. 
Tabela 1 - Produtos comerciais, doses, ingredientes ativos (IA), grupos químicos (GQ), concentrações e formulações.

\begin{tabular}{|c|c|c|c|c|c|c|}
\hline \multicolumn{2}{|c|}{ Tratamentos } & $\begin{array}{l}\text { Doses } \\
\text { L-kg.ha-1 (1) }^{-1}\end{array}$ & $\mathbf{I A}^{(2)}$ & $\mathbf{G Q}^{(3)}$ & $\begin{array}{l}\text { Concentração } \\
\text { g.L } \mathbf{L}^{-1} \text { ou g.kg } \text { kg }^{-1}\end{array}$ & Form. ${ }^{(5}$ \\
\hline 1 & Controle & - & - & - & - & - \\
\hline 2 & Engeo Pleno S & 0,25 & $\mathrm{~T}+\mathrm{L}-\mathrm{C}$ & $\mathrm{N}+\mathrm{PI}$ & $141+106$ & $\mathrm{SC}$ \\
\hline 3 & Connect & 1,00 & $\mathrm{I}+\mathrm{B}-\mathrm{C}$ & $\mathrm{N}+\mathrm{PI}$ & $100+12,5$ & $\mathrm{SC}$ \\
\hline 4 & Galil SC & 0,40 & $\mathrm{I}+\mathrm{B}$ & $\mathrm{N}+\mathrm{PI}$ & $250+50$ & $\mathrm{SC}$ \\
\hline 5 & Talstar $100 \mathrm{CE}$ & 0,30 & $\mathrm{~B}$ & PI & 100 & $\mathrm{EC}$ \\
\hline 6 & Orthene $750 \mathrm{BR}$ & 1,00 & $A+S-A$ & $\mathrm{O}$ & $750+225,5$ & SP \\
\hline
\end{tabular}

(1) L-kg.ha-1: litros ou quilograma por hectare;

(2) I.A: Ingredientes ativos; T: Tiametoxam, L-C: Lambda-Cialotrina, I: Imidacloprido, B-C: Beta-Ciflutrina, B: Bifentrina, A: Acefato, S-A: Silicato de alumínio;

(3) GQ: Grupos químicos, N: Neonicotinoide, PI: Piretroide, O: Organofosforado;

(4) g.L-1: gramas de ingrediente ativo por litro de produto comercial; g.kg-1: gramas de ingrediente ativo por quilograma de produto comercial

(5) Form: formulações, SC: suspensão concentrada, EC: concentrado emulsionável, SP: Pó Solúvel.

Fonte: Autores.

O experimento foi instalado em placas de petri, com seis tratamentos, quatro repetições e dois estágios de desenvolvimento dos insetos [ninfas (3-4 instar) e adultos]. Cada placa de petri continham dois indivíduos, totalizando em 16 percevejos por tratamento ( 8 ninfas e 8 adultos). O preparo das placas de petri foi realizado utilizando papel filtro e alimento que ficou disponível para os percevejos como: vagem de feijão, grãos de amendoim e soja. A aplicação dos produtos foi realizada com o auxílio de uma seringa adaptada às finalidades laboratoriais com um bico tipo leque, volume de calda de $150 \mathrm{~L}$ ha $^{-1}$, aplicados sobre as placas de petri, as quais continuam o substrato alimentar para os percevejos (vagens). Após a aplicação, os insetos foram alocados na placa tratada.

A avaliação do tempo letal foi realizada por um período de sete dias observando a mortalidade dos insetos. No período de 24 horas foram realizadas avaliações sequenciais, sendo que nas primeiras quatro horas foram avaliadas a cada dez minutos, nas próximas quatro horas a cada trinta minutos e nas próximas 16 horas a cada sessenta minutos. O tempo letal foi determinado pelos estimadores Kapplan-Meier e as curvas tempo x mortalidade plotadas utilizando o programa SigmaPlot.

A avaliação do período residual dos produtos foi realizada conforme as informações apresentadas na Tabela 1 . O experimento foi instalado em placas de petri, com seis tratamentos, quatro repetições, dois estágios de desenvolvimento dos insetos, sendo ninfas e adultos e seis tempos diferentes, sendo eles 0, 6, 12, 24, 48 e 72 horas. Cada placa de petri continham dois indivíduos amostrais, totalizando em 576 percevejos no experimento, 288 ninfas e 288 adultos.

O preparo das placas de petri foi realizado utilizando papel filtro e alimento que ficou disponível para os percevejos como: vagem de feijão, grãos de amendoim e soja. A aplicação dos produtos foi realizada com o auxílio de uma seringa adaptada às finalidades laboratoriais com um bico tipo leque. Após a aplicação, os insetos foram alocados na placa tratada, sendo que o tempo 0 horas, os insetos foram alocados imediatamente após a aplicação dos produtos; 6 horas, os insetos foram alocados após 6 horas de aplicação dos inseticidas; 12 horas, os insetos foram alocados após 12 horas de aplicação dos inseticidas; 24 horas, os insetos foram alocados após 24 horas de aplicação dos inseticidas; 48 horas, os insetos foram alocados após 48 horas de aplicação dos inseticidas e 72 horas, os insetos foram alocados após 72 horas de aplicação dos inseticidas.

A avaliação do período residual foi realizada após 24 horas da infestação em cada placa de petri. O tempo período residual foi determinado utilizando regressão linear $(\alpha=0,05)$ e as curvas tempo x mortalidade plotadas utilizando o programa 
SigmaPlot.

\section{Resultados e Discussão}

De forma geral, os tempos letais para causar a mortalidade em $50 \%$ da população de ninfas foram maiores do que de adultos (Figura 1, Tabela 2). O inseticida imidacloprido + bifentrina apresentou o menor tempo letal sobre ninfas de E. heros. Similarmente, para a população de adultos, esse menor tempo foi observado para o inseticida Lambda-cialotrina + Tiametoxam (Figura 1, Tabela 2). De forma conjunta, dois inseticidas apresentaram tempos similares para ambas as fases: imidacloprido + bifentrina (Ninfas $=0,456$ horas, Adultos $=0,496$ horas) e Lambda-cialotrina + tiametoxan $($ Ninfas $=0,496$ horas, Adultos $=$ 0,518 horas).

Figura 1 - Probabilidade de sobrevivência de (A) ninfas (Log-Rank Test: $\chi^{2}=60,52$, g.l. $\left.=5, \mathrm{P}<0,001\right)$ e (B) adultos (LogRank Test: $\chi 2=50,12$, g.l. $=5, \mathrm{P}<0,001)$ de Euschistus heros ao longo do tempo expostos a cinco inseticidas.

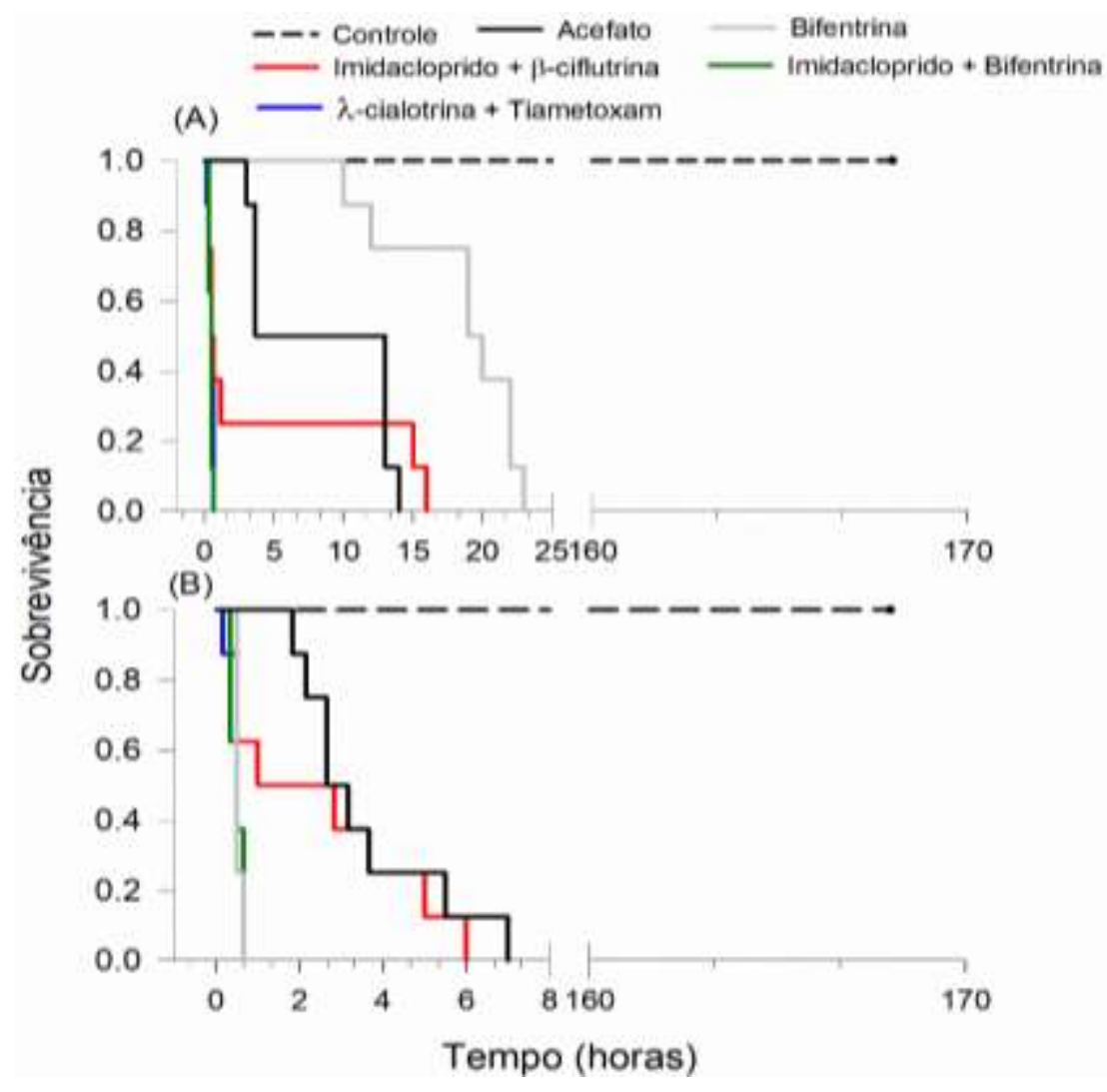

Fonte: Autores. 
Tabela 2 - Tempos letais de cinco inseticidas para causar a mortalidade em $50 \%$ da população de ninfas e adultos de Euschistus heros. Valores representam a mediana e o intervalo de confiança a $95 \%$.

\begin{tabular}{|c|c|c|}
\hline \multirow{2}{*}{ Inseticida } & \multicolumn{2}{|c|}{ TLso $( \pm I C 95 \%)$} \\
\hline & Ninfa & Adulto \\
\hline \multirow{2}{*}{ Acefato } & 8,373 & 3,579 \\
\hline & $(4,749-21,707)$ & $(2,341-4,817)$ \\
\hline \multirow{2}{*}{ Bifentrina } & 18,375 & 0,540 \\
\hline & $(15,043-21,707)$ & $(0,489-0,591)$ \\
\hline \multirow{2}{*}{ Imidacloprido $+\beta$-ciflutrina } & 4,310 & 2,499 \\
\hline & $(0,483-9,103)$ & $(0,967-4,301)$ \\
\hline \multirow{2}{*}{ Imidacloprido + Bifentrina } & 0,456 & 0,496 \\
\hline & $(0,373-0,538)$ & $(0,390-0,602)$ \\
\hline \multirow{2}{*}{$\lambda$-cialotrina + Tiametoxam } & 0,496 & 0,518 \\
\hline & $(0,373-0,619)$ & $(0,403-0,632)$ \\
\hline
\end{tabular}

Fonte: Autores.

As regressões realizadas para determinar a mortalidade dos percevejos em função do tempo após a aplicação são apresentadas na Tabela 3. Em geral, os inseticidas apresentaram uma relação linear negativa para a mortalidade causada em função do tempo após a aplicação dos tratamentos. Contudo, essa relação não foi significativa para os inseticidas $\lambda$-cialotrina + Tiametoxam e Imidacloprido + $\beta$-ciflutrina para ninfas e adultos, respectivamente (Tabela 3). Pitta et al., (2018) observaram uma redução de eficiência no controle de E. heros em diferentes populações coletadas no Estado do Mato Grosso. Neste contexto, é sugerido que há uma pressão de seleção, visto que para o controle deste inseto praga acaba-se empregando basicamente os mesmos mecanismos de ação piretróide, neonicotinóide e organofosforado.

Para a população de ninfas, Imidacloprido + bifentrina apresentou o maior período residual levando a mortalidade de aproximadamente $78 \%$ da população em um período de até 100 horas após aplicação (Figura 2A). Por outro lado, para adultos, nenhum inseticida causou mortalidade superior a $40 \%$ nesse mesmo período (Figura $2 \mathrm{~B}$ ). O fato pode estar atrelado a alta capacidade de migração deste inseto no campo, migrando para áreas não aplicadas, fragmentos de florestas, plantas de cobertura, dentre outras. A diferença entre os tempos letais para as fases do percevejo pode estar associada a incompleta formação fisiológica e morfológica das ninfas, o que pode prejudicar e limitar a atividade alimentar, uma vez que não estariam com aparelho bucal e demais estruturas completamente formadas.

De forma geral, os inseticidas Bifentrina e acefato apresentaram o menor período residual. Por exemplo, a mortalidade causada decresceu com o aumento do tempo, onde valores acima de $80 \%$ foram notados até 40 horas de exposição dos produtos. Bifentrina e acefato são produtos químicos do grupo piretróide (moduladores de canais de sódio) e organofosforado (inibidores da acetilcolinesterase), respectivamente, são produtos que agem por conta e ingestão e não sistêmico sua eficiência é reduzida ao longo dos dias após aplicação, apresentando eficiências abaixo de $70 \% 12$ dias após a aplicação, em comparação aos neonicotinóides tem uma redução de 10 a 15\% de eficiência a nível de campo (De Carvalho et. al., 2017).

Os resultados deste estudo demostram que, os inseticidas podem reduzir a população de adultos em até 40 horas, ou 2 a 3 dias após a aplicação. Em caso de re-infestações nas áreas, principalmente por adultos que se dispersam nas lavouras, os produtos aplicados não deixarão resíduo para ocasionar mortalidade significativa na população de percevejo marrom. Os inseticidas Imidacloprido + Bifentrina e Lambda-cialotrina + Tiametoxam são os mais eficientes para o controle de ninfas e adultos de E. heros. 
Tabela 3 - Sumário da análise de variância da regressão entre a mortalidade de ninfas e adultos de Euschistus heros em função do tempo após a aplicação de cinco inseticidas. * significativo quando $P<0,05$.

\begin{tabular}{lllll}
\hline Inseticida & Equação & $\mathbf{F}$ & $\boldsymbol{P}^{*}$ & $\mathbf{R}^{\mathbf{2}}$ \\
\hline Ninfa & $\mathrm{y}=114,88-1,02 \mathrm{x}$ & 17,80 & 0,01 & 0,77 \\
Acefato & $\mathrm{y}=113,68-1,08 \mathrm{x}$ & 11,48 & 0,02 & 0,67 \\
Bifentrina & $\mathrm{y}=114,53-0,77 \mathrm{x}$ & 19,10 & 0,01 & 0,82 \\
Imidacloprido + $\beta$-ciflutrina & $\mathrm{y}=117,34-0,50 \mathrm{x}$ & 24,07 & $<0,01$ & 0,82 \\
Imidacloprido + Bifentrina & $\mathrm{y}=90,98-0,43 \mathrm{x}$ & 6,75 & 0,06 & 0,53 \\
$\lambda$-cialotrina + Tiametoxam & & & & \\
Adultos & $\mathrm{y}=100,36-1,19 \mathrm{x}$ & 24,91 & $<0,01$ & 0,82 \\
Acefato & $\mathrm{y}=120,16-1,39 \mathrm{x}$ & 19,72 & 0,01 & 0,78 \\
Bifentrina & $\mathrm{y}=110,67-1,23 \mathrm{x}$ & 7,03 & 0,06 & 0,54 \\
Imidacloprido + $\beta$-ciflutrina & $\mathrm{y}=120,46-0,89 \mathrm{x}$ & 12,46 & 0,02 & 0,69 \\
Imidacloprido + Bifentrina & $\mathrm{y}=137,48-1,14 \mathrm{x}$ & 43,87 & $<0,01$ & 0,89 \\
$\lambda$-cialotrina + Tiametoxam & & & & \\
\hline
\end{tabular}

Fonte: Autores. 
Figura 2 - Mortalidade de (A) ninfas e (B) adultos de Euschistus heros em função do tempo após a aplicação de cinco inseticidas sobre o substrato alimentar. As equações e os resultados estatísticos são apresentados na tabela 3.

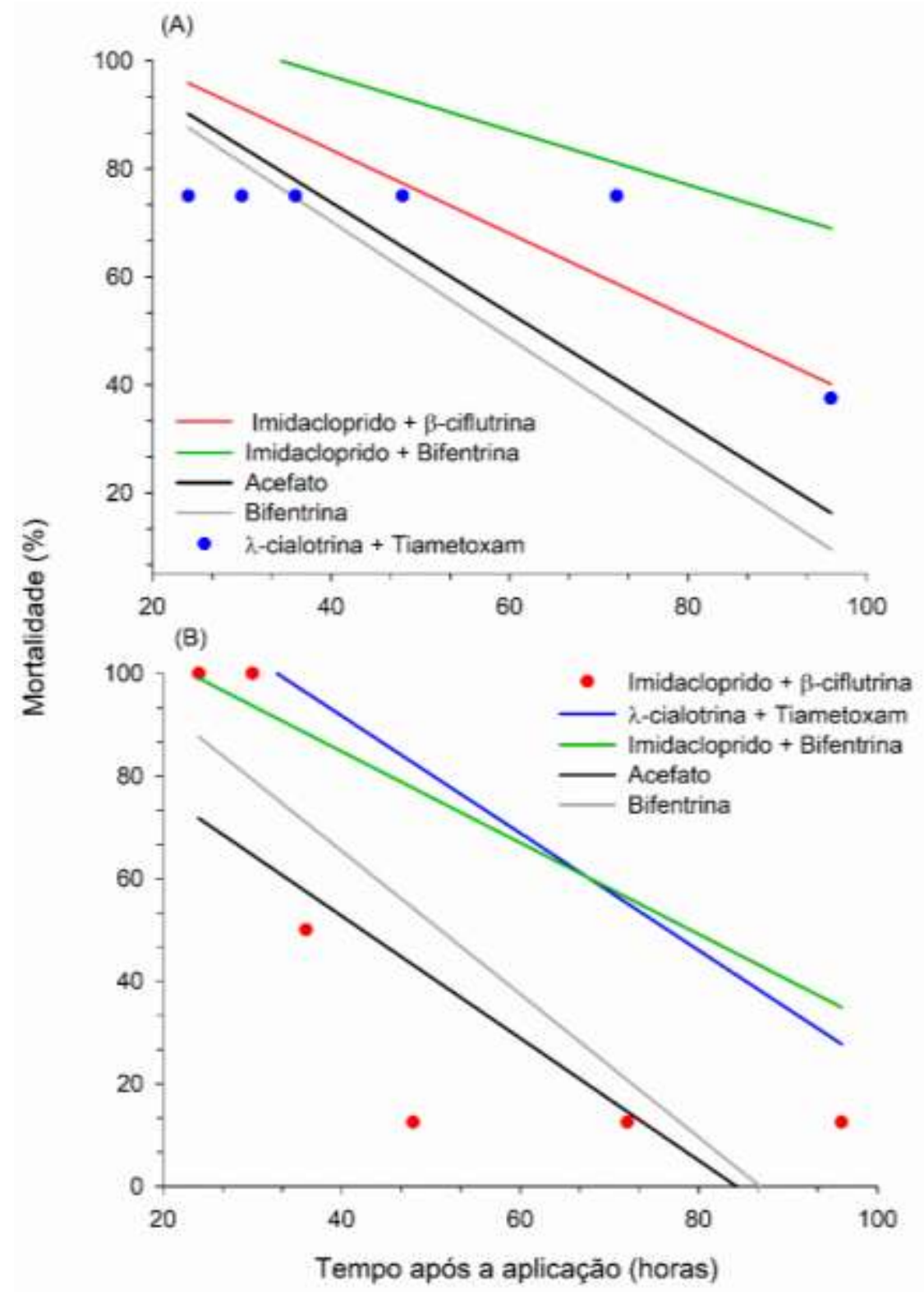

Fonte: Autores.

\section{Conclusão}

Os inseticidas Imidacloprido + Bifentrina e Lambda-cialotrina + Tiametoxam são os mais eficientes para o controle de ninfas e adultos de E. heros por apresentarem menor tempo letal e maior período residual. Futuros trabalhos são recomendados para determinar a eficiência desses produtos em condições de campo.

\section{Agradecimentos}

À CropSolutions por ceder a estrutura física e os insetos para a realização deste trabalho. 
Research, Society and Development, v. 10, n. 6, e29810616364, 2021

(CC BY 4.0) | ISSN 2525-3409 | DOI: http://dx.doi.org/10.33448/rsd-v10i6.16364

\section{Referências}

Bueno, A. F., Paula Moraes, S. V., Gazzoni, D. L., \& Pomari, A. F. (2013). Economic thresholds in soybean integrated pest management: old concepts, current adoption, and adequacy. Neotropical Entomology, 42, 439-447.

[Conab] Companhia Nacional de Abastecimento. (2020). Acompanhamento de safra brasileira: grãos, Safra 2019/2020. Nono Levantamento, Brasília. https://www.conab.gov.br/info-agro/safras/graos/boletim-da-safra-de-graos

Corrêa-Ferreira, B. S., \& Azevedo, J. (2002). Soybean seed damage by different species of stink bugs. Agricultural and Forest Entomology, 4 (2), 145-150.

Corrêa-Ferreira, B. S., Krzyzanowski, F. C., \& Minami, C. A. (2009). Percevejos e a qualidade da semente de soja - Série sementes. Londrina: Embrapa Soja.

De Carvalho, F. R., Erasmo, E. A. L., de Moura Garcia, J. P., de Farias, D. I. O. A., de Sousa Rocha, F., \& Cerqueira, F. B. (2017). Eficiência de inseticidas no controle preventivo do percevejo-marrom na cultura da soja. Revista Tecnologia e Ciência Agropecuária, 11 (1), 25-30.

Ferrazza, F. L. F., Jacoboski, D. T. K., Wyrepkowski, A., Rodrigues, L., Figueiro, A. G., \& Paraginski, R. T. (2020). Qualidade de sementes e parâmetros produtivos de soja submetidas a diferentes tratamentos de sementes antes da semeadura. Research, Society and Development, 9 (9), 1-17.

Galileo, M. H. M., \& Heinrichs, E. A. (1978). Efeito dos danos causados por Piezodorus guildinii (Westwood, 1837) (Hemiptera, Pentatomidae), em diferentes níveis e épocas de infestação, no rendimento de grãos de soja [Glycine max (L.) Merrill]. Anais da Sociedade Entomológica do Brasil, 7, 20-25.

Krinski, D., Favetti, B. M., Lima, A. G., \& Brum, T. R. (2013). Oviposition preference of the neotropical brown stink bug Euschistus heros on artificial substrates of different colors. Ciência Rural, 43 (12), 2185-2190.

Moscardi, F., Bueno, A. F., Sosa-Gómez, D. R., Roggia, S., Hoffmanncampo, C. B., Pomari, A. F., Corso, I. C., \& Yano, S. A. C. (2012). Artrópodes que atacam as folhas da soja. In: Hoffmann-Campo, C. B., Corrêa-Ferreira, B. S., \& Mosrcadi, F. (Eds.). Soja: Manejo Integrado de Pragas e outros Artrópodespraga. Brasília, DF: Embrapa, 213-309.

Panizzi, A. R., Bueno, A. F., \& Silva, F. A. C. (2012). Insetos que atacam vagens e grãos. In: Hoffmann-Campo, C. B., Côrrea-Ferreira, B. S., Moscardi, F. (Eds). Soja: Manejo Integrado de Insetos e outros Artrópodes-Praga. Brasília, DF: Embrapa, 335-420.

Panizzi, A. R. (2015). Growing problems with stink bugs (Hemiptera: Heteroptera: Pentatomidae): species invasive to U.S. and potential neotropical invaders. American Entomologist, 61, 223-233.

Pitta, R. M., Rodrigues, S. M. M., Pitta, R. M., Rodrigues, S. M. M., Vivan, L. M., \& Bianchin, K. A. (2018). Suscetibilidade de Euschistus heros (Fabr. 1794) (Heteroptera: Pentatomidae) a inseticidas em Mato Grosso. Scientific Electronic Archives, 11:1-5.

Sosa-Gómez, D. R., \& Moscardi, F. (1995). Retenção foliar diferencial em soja provocada por percevejos (Heteroptera: Pentatomidae). Anais da Sociedade Entomológica do Brasil, 24: 401-404, 1995.

Sosa-Gómez, D. R., Silva, J. J., Lopes, I. O., Corso, I. C., Almeida, A. M., Moraes, G. C., \& Baur, M. E. (2009). Insecticide susceptibility of Euschistus heros (Heteroptera: Pentatomidae) in Brazil. Journal of Economic Entomology, 102, 1209-1216.

Sosa-Gómez, D. R., \& Silva, J. J. (2010). Neotropical brown stink bug (Euschistus heros) resistance to metamidophos in Paraná, Brazil. Pesquisa Agropecuária Brasileira, 45, 767-769.

Vivan, L. M., \& Degrande, P. E. (2011). Pragas da soja. Fundação MT.

Zambiazzi, E. V., Nadai, J., Guilherme, S. R., \& Bonaldo, S. M. (2012). Controle biológico in-vitro do percevejo-marrom (Euschistus heros) com Beauveria bassiana. Revista Trópica - Ciências Agrárias e Biológicas, 6 (2), 44-48. 